\title{
How network structure shapes pairwise correlations between integrate-and-fire neurons Birgit Kriener ${ }^{* 1,2}$ and Marc Timme ${ }^{1,2}$
}

\author{
Address: ${ }^{1}$ Network Dynamics Group, Max-Planck-Institute for Dynamics and Self-Organization, D-37073 Göttingen, Germany and ${ }^{2}$ Bernstein \\ Center for Computational Neuroscience, D-37073 Göttingen, Germany \\ Email: Birgit Kriener* - kriener@nld.ds.mpg.de \\ * Corresponding author
}

from Eighteenth Annual Computational Neuroscience Meeting: CNS*2009

Berlin, Germany. 18-23 July 2009

Published: I 3 July 2009

BMC Neuroscience 2009, I0(SuppI I):PI52 doi:I0.II86/I47|-2202-I0-SI-PI52

This abstract is available from: http://www.biomedcentral.com//47I-2202/I0/SI/PI52

(c) 2009 Kriener and Timme; licensee BioMed Central Ltd.

Correlations between neurons can be caused by various mechanisms and can be pronounced on different levels on the level of spikes or membrane potentials - and both in time and space. Here, we analyze quantitatively the role of network structure on pairwise correlations in a model of local recurrent networks of excitatory and inhibitory leaky integrate-and-fire neurons. Especially correlations between the input currents of neurons prove to be highly sensitive to the statistical features of network structure like topology and weight distribution. We studied a variety of network topologies, ranging from random to small-world and regular $[1,2]$. If the external input spike trains are uncorrelated and Poissonian, input correlations between neurons are predominantly due to direct and indirect common input that is inherent to the recurrence of the local network. We speak of direct common input if two neurons share a common presynaptic neuron, and of indirect common input if two neurons receive inputs from two different presynaptic neurons, which in turn share a common presynaptic neuron.

Even in sparse balanced random networks the hard wiring of the neurons suffices to cause significant pairwise correlations of their subthreshold dynamics that are partially, but weakly transmitted to the spike level. These weak pairwise spike correlations can cause significant fluctuations of the population activity [3]. If we include a distance dependent connectivity ("spatial footprint"), common input correlations become more and more increased the more spatially confined the footprint is, given the mean number of incoming synapses per neuron is fixed. We can quantitatively describe the distance dependent correlations in a shot-noise approach and even analytically derive the full distribution of input correlation coefficients for certain cases [2].

Here we extend our former analysis to networks with multiple populations and more realistic probabilistic Gaussian footprints [4]. Moreover, we analyze how correlated topographic external inputs change the correlation structure of the local network to address the question which spatio-temporal features of external ("thalamic") feed-forward inputs enhance or also decrease pairwise correlations within the local ("cortical") recurrent network in dependence of the structural properties of the local network. Our work is aimed to eventually help separate "stimulus"-induced correlations from those that arise due to structural ("hard-wired") features alone.

\section{Acknowledgements}

We gratefully acknowledge funding by the German Federal Ministry of Education and Research (BMBF grant 0IGQ0430).

\section{References}

I. Watts DJ, Strogatz SH: Collective dynamics of small-world networks. Nature 1998, 393:440-442.

2. Kriener B, Helias M, Aertsen A, Rotter S: Correlations in spiking neuronal networks with distance dependent connections. J Comput Neurosci in press.

3. Kriener B, Tetzlaff T, Aertsen A, Diesmann M, Rotter S: Correlations and population dynamics in cortical networks. Neural Comput 2008, 20:2185-2226. 
4. Hellwig B: A quantitative analysis of the local connectivity between pyramidal neurons in layers $2 / 3$ of rat visual cortex. Biol Cybern 2000, 82:111-12I.

Publish with Bio Med Central and every scientist can read your work free of charge

"BioMed Central will be the most significant development for disseminating the results of biomedical research in our lifetime. " Sir Paul Nurse, Cancer Research UK

Your research papers will be:

- available free of charge to the entire biomedical community

- peer reviewed and published immediately upon acceptance

- cited in PubMed and archived on PubMed Central

- yours - you keep the copyright

Submit your manuscript here:

http://www.biomedcentral.com/info/publishing_adv.asp 